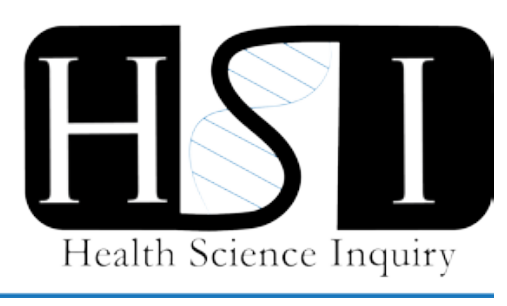

\title{
A call for interdisciplinary collaboration between video game designers and health care professionals to fight obesity
}

\begin{abstract}
Shahan Salim, Michael Lukacs
Faculty of Health Sciences, University of Western Ontario

More than $26 \%$ of Canadian adults are obese and $12 \%$ of school-aged children are overweight or obese; together, this accounts for an annual health care cost of $\$ 3.9$ billion in Canada [1]. Obesity contributes to the early development of a number of diseases such as type 2 diabetes, osteoarthritis, colorectal cancer, depression and premature death [1]. Some commonly reported barriers to regular physical activity include lack of time, social support, space to exercise, transportation and funds to buy equipment or join facilities [2].
\end{abstract}

Ubiquitous and accessible consumer technologies can assist people struggling with obesity to recover and maintain a good quality of life. This is especially true for the new generation of video games-"exergames" - that require exertion in the form of strength training, balance and flexibility activities. Exergames can offer the combination of the benefits of physical activity and the entertaining and motivating factors of video games. They have the potential to increase accessibility and affordability of treatment options while reducing the strain on the health care system. Video games are intrinsically motivating - people engage with them because they are inherently interesting and are designed to be enjoyable [3]. However, commercial exergames have been unable to replicate the recommended exertion requirements as they are primarily designed to maximize entertainment [4]. Video games that are tailormade for health, on the other hand, lack the graphics and gameplay standards of commercial games [5] and risk losing user interest. The loss in interest is partly due to the fact that tailor-made video games are based on extrinsic motivation. People engage with them because they lead to desirable but separable outcomes which are achieved over time, resulting in loss of interest as results are delayed [3]. Action void of intrinsic motivation will result in failure of any activity-based intervention. Effective game design must include interdisciplinary collaboration between healthcare professionals and video game designers for exergames to be a successful treatment option.

Research on exergames has produced mixed results with variability in data based on the type of game, time spent playing each game, and weight and age of participants. Exergaming results in higher levels of energy expenditure in both adults and children, and higher energy expenditure than in heart sedentary video games [6]. Respiratory exertion while exergaming exhibited respiratory efforts that were comparable to medium intensity aerobic dance [7] and energy expenditure and heart rate similar to brisk walking or jogging [8]. However, the amount of energy expenditure during exergaming is not comparable to the recommended amounts of daily physical activity. Energy expenditure during exergaming falls below the recommended levels for maintaining cardio-respiratory fitness [6]. Exergames also have low half-lives as users get tired of the game and move on to more exciting sedentary videogames; users engaged in exergaming for 6 minutes compared to at least 60 minutes of physical activity per day as recommended by health agencies [7].

Gamification is defined as using game design elements in a non-game context and can be the solution to increase intrinsic motivation in exergames. To understand "fun" in video games, researchers have used the Self Determination Theory (SDT), which emphasizes motivation to perform a behavior (9). Motivation has been identified as the key characteristic for enjoying game play, and enjoyment of engaging in a certain behavior is an important characteristic of motivation. Enjoyment is a function of feeling autonomous (the perception of making in-game choices), competent (being good at the game) and reliable (perception of relating to personal values). The concepts of attractiveness, motivation and incentive are also similar to the concept of Utility Value in the field of economics (10) that quantifies a person's satisfaction from consuming a good or service but emphasizes that a consumer's utility value is difficult to measure and changes from person to person. Based on these principles of gamification it should be noted that (i) exergame as a motivational tool is not designed for people who already engage in traditional physical activity, (ii) there will not be a onesize-fits-all solution and (iii) effectiveness of exergames is a combination of the motivational and physical benefits (10). 
Therefore the crux of the issue is that while exergames can be an effective tool to fight obesity, the sports and training science aspects play a secondary role in the design and development process of these games [4]. It is clear that future exergames design and production require significant contribution from health care professionals. In turn health care professionals must concede that exergame design will include addictive elements to intrinsically motivate users. Effective exergames must balance recommendations from physicians and physiotherapists on variables such as the types of exercise, duration, range of motion and speed, while video game designers make the game fun and addictive. The success of exergames can only be achieved through interdisciplinary collaboration between video game designers and health care professionals.

\section{References}

[1] Janssen, I., (2013) The Public Health Burden of Obesity in Canada. Canadian Journal of Diabetes, 37, 9096.

[2] Forkan, R., Pumper, B., Smyth, N., Wirkkala, H., Ciol, M. A., Shumway-Cook, A. (2006) Exercise Adherence Following Physical Therapy Intervention in Older Adults With Impaired Balance. Phys Ther., 86 (3), 401410.

[3] Birk, M. V., Mandryk, R. L., Atkins, C. (2018) The Motivational Push of Games: The Interplay of Intrinsic Motivation and External Rewards in Games for Training. Available from: http://hci.usask.ca/uploads/396avatarCHIPlay2016_camera_ready.pdf.

[4] Hardy, S., Göbel, S. (2012) Adaptation Model for Indoor Exergames. Int J Comput Sci Sport,
11 (1), 7385. Available from: ftp://www.kom.tudarmstadt.de/papers/HGG+11-4.pdf.

[5] Kato, P. M. (2018) Video Games in Health Care: Closing the Gap. Available from: https://www.apa.org/pubs/journals/releases/gpr14-2-113.pdf.

[6] Graves, L., Stratton, G., Ridgers, N. D., Cable, N. T. (2007) Comparison of energy expenditure in adolescents when playing new generation and sedentary computer games: cross sectional study. BMJ, 335 (7633), 12821284.

[7] Tan, B., Aziz, A. R., Chua, K., Teh, K. C. (2002) Aerobic demands of the dance simulation game. Int $J$ Sports Med., 23 (2), 125129.

[8] Maddison, R., Mhurchu, C. N., Jull, A., Jiang, Y., Prapavessis, H., Rodgers, A. (2007) Energy expended playing video console games: an opportunity to increase childrens physical activity? Pediatr Exerc Sci., 19 (6), 334343.

[9] Mellecker, R., Lyons, E. J., Baranowski, T. (2013) Disentangling Fun and Enjoyment in Exergames Using an Expanded Design, Play, Experience Framework: A Narrative Review. Games Health J, 2 (3), 142149. Available from: http://online.liebertpub.com/doi/abs/10.1089/g4h.201 3.0022

[10] Whitehead, A., Johnston, H., Nixon, N., Welch, J. (2010) Exergame effectiveness: what the numbers can tell us. Proc 5th ACM SIGGRAPH Symp Video Games, 7AD, 5562.

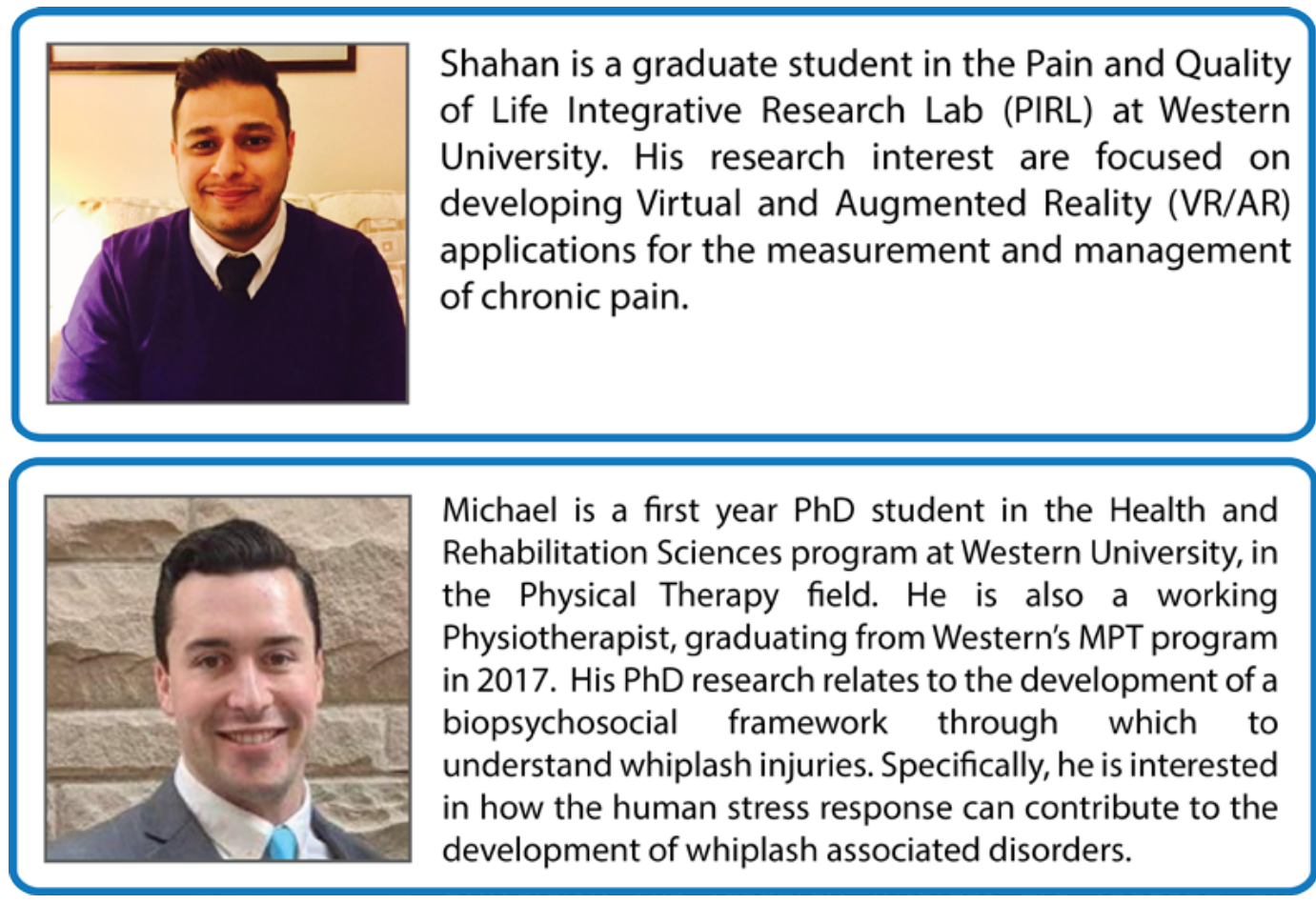

